These are the single nebula hypotheses of Kant (1755) and Laplace (1796), the accretion theory of Berkeland (1912), the exploding binary component model originally proposed by Gunn (1932) and the two-star tidal filament picture first proposed by Buffon (1745). Each has been fully articulated by modern physicists (Hoyle, for example, has contributed to all of them), who have introduced much inventive detail. The earlier chapters of this book, written by authorities such as Cameron, McCrea, Woolfson and Prentice provide wellwritten accounts of particular modern variants of these theories. Each is able to account, more or less, for the principal features of the Solar System: the coplanarity of planetary orbits, the contrary imbalances of mass and angular momentum between Sun and planets, the Titius-Bode orbit relationship, and planetary chemical compositions. Each such chapter is well-reasoned and persuasive, with the exception of an irritatingly dogmatic essay by Alfvén, ostensibly critical of myth in science, but guilty of more unsupported assumption that any of the theories criticised.

Considered as a snapshot of the way the subject looked in 1976, and as a wideranging introduction to various aspects of the subject for students with a physics background, the book has much to recommend it. It is also a valuable casestudy of the Popperian dictum that a necessary criterion for judging a scientific theory is its experimental or observational falsifiability. Although much of the theoretical work presented yields predictions which are falsifiable in principle, the authors must be aware that they are safe in practice, at any rate for the next few years. Only time and improved instrumentation will reveal the extent of their self-indulgence.

John Beckman

J. E. Beckman is in the Department of Physics at Queen Mary College, University of London, UK.

\section{Cancer for the layman}

The Wayward Cell. Cancer: Its Origins, Nature, and Treatment. Second edition. By V. Richards. Pp. 407. (University of California Press: Berkeley, Los Angeles and London, 1978). £8.75.

The Wayward Cell is a most interesting and unique treatise on various aspects of oncological diseases, their origins, nature and clinical care. It aims to provide facts and figures about cancer which are easily understandable to the intelligent layman as well as those involved with paramedical

\section{Selected immunological methods}

Immunological Methods. Edited by I. Lefkovits and B. Pemis. Pp.488. (Academic, New York and London, 1979.) $\$ 36 ; £ 23.40$.

Immunological Methods is a misleading title for this volume of techniques. A nonimmunologist hoping to find recipes for standard immunolgical methods, such as immunodiffusion in agar and immunoelectrophoresis, will be disappointed. A better title would be: Experimental Techniques used by Scientists at the Basle Institute for Immunology. This title, although slightly long for the spine of the book, would justify the inclusion of the chapters on nanomole peptide mapping (B.A. Moss, chapter 3 ), the production of sendai virus disciplines. However, I believe that this book will also interest and stimulate clinical oncologists, both those who are established and those in training.

The idea for the book almost certainly was born from the frustrations which clinicians not infrequently experience both with respect to their own failure to cure some tumours and with the need to provide clear concise explanations of the protean manifestations of cancer often sought by their patients.

This volume is divided into four main sections dealing with the biology, clinical aspects and treatment of cancer and some of the social problems it causes. The biology part is concerned with aspects of cell
(R. Tees, chapter 30), the running of an isotope laboratory (H.M. Pohlit et al., chapter 33) and the excellent introduction to polyacrylamide slab gel electrophoresis (B. Takacs, chapter 4), all of which can hardly be described as immunological methods.

The editors have pursued a policy of allowing individual contributors freedom of style and content. This approach has resulted in a variation in depth of treatment afforded different topics and an emphasis on murine cellular immunology. For example, serological typing of human histocompatibility antigens is briefly treated in 10 pages (J.W. Stocker and D. Bernoco, chapter 14). An assay for specific alloantigen binding $T$ cells activated in the murine mixed lymphocyte reaction is treated in detail and clarity in 17 pages (Elliott et al., chapter 21).

Another problem resulting from the lack of editorial direction is the repetition of several techniques in different chapters, the most exteme example of which is the description of suspension cloning growth and differentiation together with modern views of the importance of molecular biology in enabling us to understand how cells work.

The clinical section is perhaps the best part of the book with a fascinating and informative account of the history of cancer. The importance of exposure to environmental carcinogens in causing the numerous cancers of this day and age is dealt with logically and clearly together with a simple account of the ways in which such carcinogens might influence cell growth and function. It is perhaps a pity that this section contains no simple pathological classification of the various types of tumours to illustrate their diversity of structure and histogenesis.

The section on treatment will be of particular value to the non-medical person giving, albeit with perhaps undue emphasis on breast cancer, the background to the various therapeutic modalities in current use.

The final part of the book is called "Cancer - 1977" and is an attempt to present the current state of our knowledge, its deficiencies and the prospects for the future. This section is disappointing, being too detailed and scientific for the lay reader. Elements would have been better integrated with the other sections.

Overall, this is a good book, well written and excellently illustrated with clinical case reports to reveal the patient problems which tumours create. Very few books of this nature are available with such a wealth of information. It should interest the range of readers for which it is intended.

A. Munro Neville

A Munro Neville is at the Ludwig Institute for Cancer Research, Royal Marsden Hospital, Sutton, UK. techniques for lymphoid cells in three chapters (M. Shulman, chapter 20; N.N. Iscove and M.H. Schreier, chapter 29; G. Köhler, chapter 32).

Among the better chapters in the volume is a delightfully written account of antibody binding characteristics (S. Fazekas de of Groth, chapter 1). Another bonus is the presence of techniques for making monoclonal antibodies (G. Kohler, chapter 31), although this latter chapter would have been more valuable if it had been updated to include PEG fusion techniques and extended to include different screening techniques.

This book can be commended to specialist immunologists, particularly cellular immunologists, but does not serve as an introduction to immunological techniques for the non-specialist.

Peter Goodfellow

Peter Goodfellow is a staff scientist at the Imperial Cancer Research Fund's Laboratories, London, UK. 